\title{
Hemogasometria arterial pré e pós-rinoplastia em cães braquicefálicos portadores de estenose de narina
}

[Arterial hemogasometry pre and post rhinoplasty in brachyphalic dogs with nostril stenosis]

\author{
A.F. Mendes Junior ${ }^{1}$, G.S.O.D. Silva ${ }^{2}$, V.M. Silva ${ }^{2}$, S.C.G. Silva ${ }^{2}$, S.P. Braga ${ }^{2}$, \\ C.R. Leite ${ }^{3}$, A.M.B. Soares ${ }^{3}$, N.R.P. Almosny ${ }^{3}$ \\ ${ }^{1}$ Aluno de pós-graduação - Universidade Federal Fluminense - Niterói, RJ \\ ${ }^{2}$ Aluno de graduação - Universidade Federal Fluminense - Niterói, RJ \\ ${ }^{3}$ Universidade Federal Fluminense - Niterói, RJ
}

\begin{abstract}
RESUMO
Vinte e seis cães braquicefálicos portadores de estenose de narina, 22 machos e quatro fêmeas, foram submetidos à rinoplastia bilateral. Dezesseis cães eram Buldogues Franceses; dois, Buldogues Ingleses; seis, Pugs; e dois, Shih Tzus, com idade variando de seis meses a seis anos. Foram efetuadas coletas de sangue arterial para análises hemogasométricas no pré-operatório e 30 dias após a cirurgia. Para cada avaliação, foi obtida uma amostra de $0,5 \mathrm{~mL}$ de sangue coletado da artéria femoral, em seringa plástica heparinizada. Em seguida, procedeu-se à avaliação hemogasométrica em analisador de gases sanguíneos (I-stat-Abbot $\left.{ }^{\circledR}\right)$. Os resultados da hemogasometria pré e pós-rinoplastia mostraram uma redução nos valores médios de $\mathrm{pCO}_{2}, \mathrm{TCO}_{2}, \mathrm{HCO}_{3}$ - e BEecf, hematócrito e hemoglobina, e aumento de $\mathrm{pH}, \mathrm{pO}_{2} \mathrm{e}$ $\mathrm{SO}_{2}$, indicando melhora na condição ventilatória dos animais após a correção cirúrgica da estenose de narina. Isso posto, conclui-se que a hemogasometria arterial é um exame importante no diagnóstico da síndrome respiratória dos cães braquicefálicos, e extremamente útil no acompanhamento da resposta do paciente ao tratamento. A rinoplastia mostrou-se eficaz no tratamento da síndrome respiratória, promovendo melhora nos parâmetros hemogasométricos que indicam acidose respiratória secundária à obstrução das vias aéreas, comum nas raças braquicefálicas.
\end{abstract}

Palavras-chave: síndrome respiratória, acidose respiratória, prolongamento de palato mole

\begin{abstract}
Twenty-six brachycephalic dogs with nostril stenosis, 22 males and four females, underwent bilateral rhinoplasty. Sixteen dogs were French Bulldogs; two, English Bulldogs; Six, Pugs; and two, Shih tzus, ranging in age from six months to six years. Blood samples were collected for hemogasometric analysis in the preoperative period and 30 days after surgery. For each evaluation, a $0.5 \mathrm{ml}$ sample of blood collected from the femoral artery was obtained in a heparinized plastic syringe. Hemogasometric evaluation was then performed on a blood gas analyzer (I-stat-Abbot $囚)$. The results of hemogasometry before and after rhinoplasty showed a reduction in the mean values of $\mathrm{pCO}_{2}, \mathrm{TCO}_{2}, \mathrm{HCO} 3$ - and $\mathrm{BEecf}$, hematocrit and hemoglobin, and an increase in $\mathrm{pH}, \mathrm{pO}_{2}$ and $\mathrm{SO}$, indicating an improvement in the ventilatory condition of the animals after surgical correction of Nostril stenosis. Therefore, it is concluded that arterial hemogasometry is an important diagnostic tool for the diagnosis of brachycephalic respiratory syndrome and is extremely useful in monitoring the patient's response to treatment. Rhinoplasty was effective in the treatment of respiratory syndrome, promoting improvement in hemogasometric parameters that indicate respiratory acidosis secondary to airway obstruction, common in the brachycephalic races.
\end{abstract}

Keywords: respiratory syndrome, respiratory acidosis, soft palate elongation

Recebido em 24 de julho de 2017

Aceito em 16 de fevereiro de 2018

E-mail: aguinaldo_zootec@hotmail.com 


\section{INTRODUÇÃO}

A síndrome respiratória dos braquicéfalos pode ser observada em cães e gatos de focinho curto. É causada por alterações anatômicas que provocam importante obstrução das vias aéreas superiores, impedindo o fluxo adequado do ar até os pulmões. Em cães, as alterações classificadas como primárias são estenose de narina, prolongamento de palato mole e hipoplasia de traqueia. Essas deformidades podem gerar alterações secundárias, como eversão de sáculos laríngeos, edema e inflamação de nasofaringe, paralisia e colapso de laringe (Meola, 2013; Dupré e Heidenrich, 2016).

Os sinais clínicos causados pela obstrução das vias aéreas são ronco, estertor, estridor, dispneia, intolerância ao exercício, cianose, dificuldade de recuperação pós-exercícios, intermação, perturbações do sono e alterações gastrointestinais e cardíacas. Esses sinais apresentam grande potencial deletério à qualidade de vida dos animais e, em muitos casos, alto risco de óbito (Packer e Tivers, 2015; Dupré e Heidenrich, 2016).

O tratamento consiste na correção cirúrgica das anormalidades anatômicas que provocam a obstrução das vias aéreas, associada ao suporte terapêutico medicamentoso, objetivando diminuir a inflamação e o edema das vias aéreas e controlar os sinais gastrointestinais (Trappler e Moore, 2011). Quanto mais anterior a resistência à entrada do ar, maior o esforço inspiratório; a estenose de narina é o estreitamento mais anterior da síndrome (Koch et al., 2003).

A hemogasometria é um exame laboratorial que auxilia no diagnóstico de inúmeras disfunções respiratórias (Roels et al., 2015). Dessa forma, torna-se extremamente útil na avaliação da resposta ao tratamento em cães braquicefálicos portadores da síndrome, visto que estes podem desenvolver, secundariamente à obstrução das vias aéreas superiores, quadros de hipóxia, que, por sua vez, leva à acidose respiratória. Diante disso, objetivou-se, com o presente estudo, avaliar valores de gases sanguíneos em cães braquicefálicos, portadores de estenose de narina, mediante a realização de hemogasometria arterial antes e depois da realização da rinoplastia, a fim de avaliar a eficácia do procedimento cirúrgico como tratamento e da hemogasometria no acompanhamento póscirúrgico.

\section{MATERIAL E MÉTODOS}

Este projeto foi aprovado pela Comissão de Ética no Uso de Animais da Universidade Federal Fluminense, sob o número de protocolo 686/2015.

Foram incluídos no estudo e submetidos à rinoplastia 26 cães braquicefálicos portadores de estenose de narina, sendo 22 machos e quatro fêmeas. Destes, 16 eram da raça Buldogue Francês; dois, da Buldogue Inglês; seis, da Pug; e dois, da Shih Tzu, com idade variando de seis meses a seis anos, e peso médio de $12,7 \mathrm{~kg}$, atendidos no Hospital Universitário de Medicina Veterinária Firmino Mársico Filho, da Universidade Federal Fluminense (Huvet-UFF), no período de março de 2015 a março de 2016. Os animais foram incluídos após assinatura pelos responsáveis de termo de consentimento livre e esclarecido.

Todos os animais passaram por exame físico (auscultação cardiopulmonar, avaliação de tempo de preenchimento capilar, temperatura corporal, grau de hidratação, avaliação de coloração de mucosas) e exames pré-operatórios (hemograma com pesquisa de hemoparasitos e microfilária, dosagem bioquímica sérica, eletrocardiograma e ecocardiograma, além de radiografia torácica em momento de inspiração e expiração, nas posições lateral direita e esquerda e ventrodorsal). Os cães que apresentaram os exames físico e complementares sem alterações foram encaminhados à rinoplastia.

Para o protocolo anestésico, utilizou-se, como medicação pré-anestésica (MPA), nalbufina, na dose de $0,5 \mathrm{mg} / \mathrm{kg}$, por via intramuscular. $\mathrm{Na}$ indução, o animal recebeu propofol $3 \mathrm{mg} / \mathrm{kg}$ e midazolam $0,3 \mathrm{mg} / \mathrm{kg}$ intravenoso. A manutenção foi realizada com isoflurano $1,5 \%$. Durante o transcirúrgico, foi administrado tramadol intravenoso, na dose de $2 \mathrm{mg} / \mathrm{kg}$ para analgesia, ceftriaxona $30 \mathrm{mg} / \mathrm{kg}$ para antibioticoterapia preventiva e dexametasona $0,5 \mathrm{mg} / \mathrm{kg}$ com função anti-inflamatória.

Para as análises hemogasométricas, foram efetuadas coletas de sangue arterial nas fases préoperatória, imediatamente antes à administração 


\section{Hemogasometria arterial...}

da medicação pré-anestésica e 30 dias após a cirurgia. Para cada avaliação, foi obtida uma amostra de $0,5 \mathrm{~mL}$ de sangue coletado da artéria femoral, em seringa plástica contendo heparina liofilizada com lítio, para avaliação hemogasométrica em analisador de gases sanguíneos (I-stat-Abbot). Os parâmetros obtidos por meio da hemogasometria foram $\mathrm{pH}$ (potencial hidrogeniônico), $\mathrm{pCO}_{2}$ (pressão parcial de dióxido de carbono), $\mathrm{pO}_{2}$ (pressão parcial de oxigênio), BEecf (déficit de bases), $\mathrm{HCO}_{3}$ (bicarbonato), $\mathrm{TCO}_{2}$ e $\mathrm{SO}_{2}$ (saturação de oxigênio).
Os pacientes foram posicionados em decúbito ventral. Com o auxílio de uma pinça BrownAdson, foi realizada uma incisão em forma de "V" ao redor da pinça, com lâmina de bisturi número 11. A cunha tecidual foi removida $\mathrm{e}$ reaproximada à área incisionada com suturas interrompidas simples com fio absorvível sintético multifilamentado (Poliglactina 910, 3-0 ou 4-0). O procedimento foi repetido no lado oposto, com o cuidado de excisar a cunha do mesmo tamanho (Fig. 1 e 2) (Fossum, Dupré, 2005).

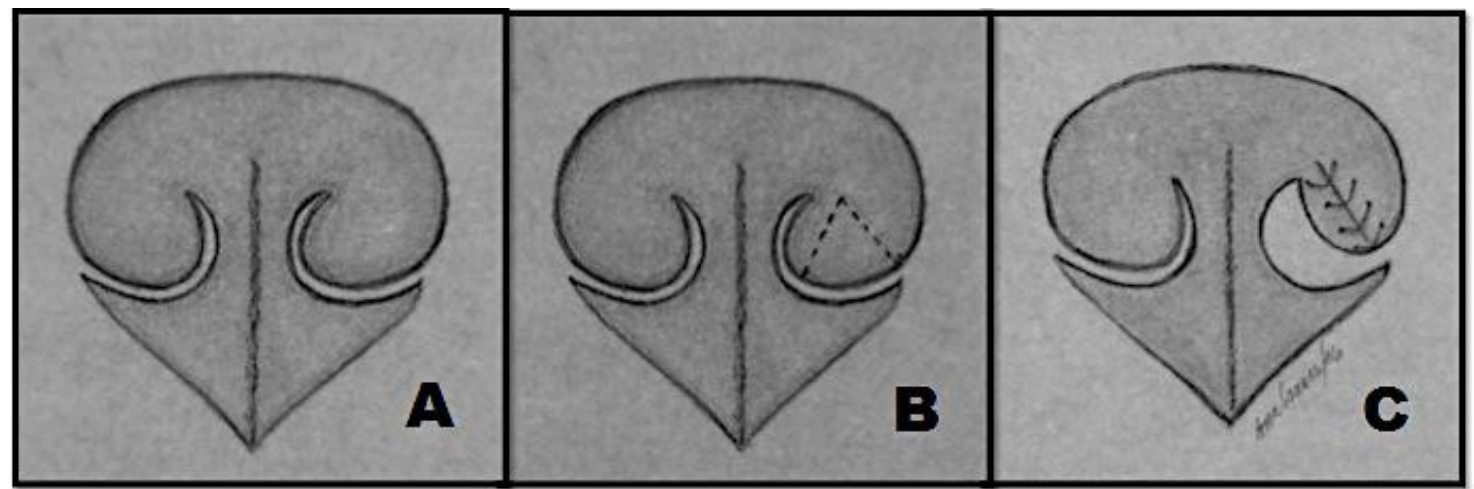

Figura 1. Ilustração da técnica de ressecção em cunha para realização de rinoplastia. Em A, narinas estenosadas; em B, marcação prévia para posterior exérese; em C, aspecto da narina após sutura em pontos simples.

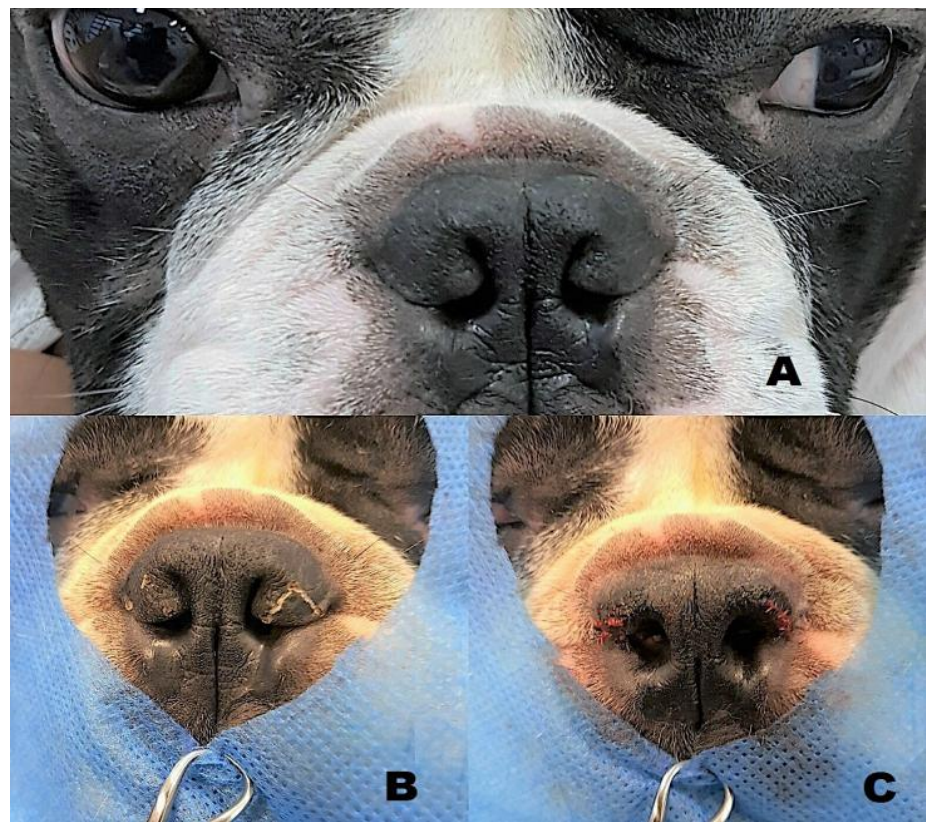

Figura 2. Buldogue Francês, macho, dois anos de idade, portador de estenose de narina. Em A, narinas estenosadas; em B, marcação prévia para posterior exérese; em $\mathrm{C}$, aspecto das narinas após sutura em pontos simples. 
Os resultados foram tabelados em planilhas do programa Microsoft Excel® e analisados com o programa estatístico SAS University Edition®. Os dados obtidos das variáveis quantitativas $(\mathrm{pH}$, $\mathrm{pCO}_{2}, \mathrm{HCO}_{3^{-}}, \mathrm{TCO}_{2}, \mathrm{SO}_{2}$ ) foram submetidos à Análise de Variância (ANOVA), e as médias entre tratamentos comparadas utilizando-se o teste $\mathrm{F}$ a $5 \%$ de significância, para verificar se houve ou não efeito de tratamento.

\section{RESULTADOS}

Os resultados da hemogasometria pré e pósrinoplastia mostraram uma redução nos valores médios de $\mathrm{pCO}_{2}$ (de 34,8269 para 33,0038),
$\mathrm{TCO}_{2}$ (de 20,9231 para 20,7308), $\mathrm{HCO}_{3^{-}}$(de 19,8615 para 19,7231 ) e BEecf (de $-5,2692$ para -4,9231), assim como um aumento de $\mathrm{pH}$ (de 7,3645 para 7,3829), $\mathrm{pO}_{2}$ (de 80,5769 para 84,653) e $\mathrm{SO}_{2}$ (de 95,3846 para 96,1154 ) (Tab. 1). A análise estatística das médias das variáveis mostrou uma diferença significativa em $\mathrm{pH}$, $\mathrm{pCO}_{2}, \quad \mathrm{pO}_{2}$ e $\mathrm{SO}_{2}$; não houve diferença estatisticamente expressiva em $\mathrm{TCO}_{2}, \mathrm{HCO}_{3}$ - e BEecf (Tab. 1).

Também foi constatada uma diminuição nas médias de hematócrito e hemoglobina, para esses parâmetros, porém a análise de variância não foi significativa.

Tabela 1. Análise descritiva e comparação das médias das variáveis $\mathrm{pH}, \mathrm{pCO}_{2}, \mathrm{pO}_{2}, \mathrm{SO}_{2}, \mathrm{TCO}_{2}, \mathrm{HCO}_{3^{-}}$, BEecf, hematócrito e hemoglobina pelo teste $\mathrm{F}$ a $5 \%$ de significância da hemogasometria arterial dos 26 cães braquicefálicos portadores de estenose de narina, antes e 30 dias depois de serem submetidos à rinoplastia

\begin{tabular}{ccccccccccc}
\hline & \multicolumn{4}{c}{ Pré-rinoplastia } & \multicolumn{4}{c}{ Pós-rinoplastia } \\
\hline Variável & $\mathrm{N}$ & Média & $\begin{array}{c}\text { Desvio } \\
\text { padrão }\end{array}$ & Mínimo & Máximo & $\mathrm{N}$ & Média & $\begin{array}{c}\text { Desvio } \\
\text { padrão }\end{array}$ & Mínimo & Máximo \\
$\mathrm{pH}$ & 26 & $7,3645 \mathrm{~b}$ & 0,0294 & 7,321 & 7,442 & 26 & $7,3829 \mathrm{a}$ & 0,0306 & 7,326 & 7,449 \\
$\mathrm{pCO}_{2}$ & 26 & $34,8269 \mathrm{a}$ & 3,5320 & 28,2 & 41,5 & 26 & $33,0038 \mathrm{~b}$ & 2,3114 & 28,8 & 38,9 \\
$\mathrm{pO}_{2}$ & 26 & $80,5769 \mathrm{~b}$ & 5,6226 & 69 & 90 & 26 & $84,6538 \mathrm{a}$ & 7,2880 & 67 & 97 \\
$\mathrm{BEecf}$ & 26 & $-5,2692 \mathrm{a}$ & 2,0699 & -9 & -2 & 26 & $-4,9231 \mathrm{a}$ & 1,6229 & -8 & -2 \\
$\mathrm{HCO}_{3^{-}}$ & 26 & $19,8615 \mathrm{a}$ & 2,1296 & 14,6 & 23,2 & 26 & $19,7231 \mathrm{a}$ & 1,4478 & 16,3 & 23 \\
$\mathrm{TCO}_{2}$ & 26 & $20,9231 \mathrm{a}$ & 2,3311 & 15 & 24 & 26 & $20,7308 \mathrm{a}$ & 1,5377 & 17 & 24 \\
$\mathrm{SO}_{2}$ & 26 & $95,3846 \mathrm{~b}$ & 1,0612 & 93 & 97 & 26 & $96,1154 \mathrm{a}$ & 1,0325 & 93 & 97 \\
Hematócrito & 26 & $46,4231 \mathrm{a}$ & 6,2301 & 32 & 55 & 26 & $44,8077 \mathrm{a}$ & 5,5138 & 35 & 53 \\
Hemoglobina & 26 & $15,7885 \mathrm{a}$ & 2,1210 & 10,9 & 18,7 & 26 & $15,2500 \mathrm{a}$ & 1,9096 & 11,9 & 18 \\
\hline
\end{tabular}

Médias seguidas de letras iguais (A) na mesma linha não são estatísticamente diferentes; médias seguidas de letras diferentes (A e B) na mesma linha apresentam diferença estatisticamente relevante.

No presente estudo, após a rinoplastia, foi observada uma diminuição das médias de $\mathrm{HCO}_{3-}$, parâmetro importante para avaliação da compensação em casos de acidose respíratória. Por fim, observou-se um decréscimo dos valores de excesso de bases (BEecf) da média de volume globular e da hemoglobina dos 26 cães avaliados.

\section{DISCUSSÃO}

A $\mathrm{pCO}_{2}$, o $\mathrm{pH}$ e o $\mathrm{HCO}_{3^{-}}$são os principais parâmetros de avaliação do componente respiratório. Em casos de acidose respiratória secundária à obstrução das vias aéreas, a produção de dióxido de carbono é maior que a excreção pelos pulmões, aumentando a $\mathrm{pCO}_{2} \mathrm{e}$ a concentração de $\mathrm{H}+$, o que leva à diminuição do $\mathrm{pH}$ e ao aumento compensátório de $\mathrm{HCO}_{3}$ (Dibartola, 2006). Assim sendo, com a redução da $\mathrm{pCO}_{2}$, do $\mathrm{HCO}_{3^{-}}$e com a elevação do $\mathrm{pH}$, após a rinoplastia dos 26 cães braquicefálicos, houve melhora na condição ventilatória dos animais após a correção cirúrgica da estenose de narina.

A redução nos valores de $\mathrm{pCO}_{2} \mathrm{e}$ o aumento de $\mathrm{pH}$ também foram dados encontrados por Slawata et al. (2011), que analisaram, em estudo semelhante ao atual, parâmetros gasométricos de sangue arterial para avaliação de distúrbios ácido-base em cinco Buldogues Franceses antes e 30 dias após a correção de estenose bilateral de narinas. Tal estudo demonstrou melhora na capacidade de ventilação desses animais póscirurgia, reforçando a eficácia da rinoplastia no tratamento da síndrome braquicefálica, fato que também foi levantado em 2015 por Slawata et al., que avaliaram a função respiratória de 60 cães adultos da raça Boxer com prolongamento e espessamento de palato mole, diagnosticados por 
endoscopia antes e após estafilectomia, e afirmaram que a intervenção cirúrgica é o tratamento de eleição para a síndrome respiratória dos cães braquicefálicos e tem como objetivo a desobstrução das vias aéreas, facilitando o fluxo de ar e, consequentemente, proporcionando melhora no processo de ventilação.

Um outro fato encontrado no estudo atual, que sugeriu a melhora ventilatória dos cães braquicefálicos submetidos à rinoplastia, foi o aumento estatisticamente significativo nos níveis médios de $\mathrm{SO}_{2}$ e $\mathrm{pO}_{2}$.

Uma substancial elevação comparativa no pré e no pós-cirurgico na $\mathrm{pO}_{2}$ (de 73,00 para 82,00) também foi relatada por Slawata et al. (2011) em estudo semelhante. Sabe-se que cães braquicefálicos apresentam uma tendência a um déficit no mecanismo de oxigenação em razão da obstrução causada pelas alterações anatômicas (Hoareau et al., 2012; Yamini et al., 2015).

No estudo atual, apesar de não significativa, observou-se uma diminuição nas médias de volume globular e hemoglobina após a rinoplastia dos 26 cães, o que também foi averiguado por Hoareau et al. (2012), que, ao compararem valores de hemoglobina em cães braquicefálicos e não braquicefálicos, encontraram valores médios maiores de hemoglobina nos cães de focinho curto $(16,7 \mathrm{mg} / \mathrm{dL})$ do que nos cães de focinho alongado $(15,2 \mathrm{mg} / \mathrm{dL})$. Os autores observaram, ainda, que esse fato era mais marcante individualmente em animais com idade mais avançada, o que seria justificado pela progressão da doença com o passar do tempo. Yamini et al. (2015) encontraram valores mais altos de volume globular em cães braquicefálicos. Cães braquicefálicos apresentam frequentemente quadros de apneia do sono, o que causa importante hipóxia, levando ao estímulo para maior produção de eritropoietina e, consequentemente, maior produção de hemácias, com o objetivo de manter normalizada a oxigenação arterial.

Observou-se, ainda, no presente estudo, um decréscimo nos valores de excesso de bases (BEecf), de -5,26 para -4,92, este também sem significância estatística. Hoareau et al. (2012) encontraram alteração em íons $\mathrm{H}+$, o que indica envolvimento de mecanismos metabólicos compensatórios no processo de acidose respiratória comum nos cães braquicefálicos.

\section{CONCLUSÃO}

Após análise dos gases sanguíneos pré e póstratamento cirúrgico, constatou-se que a rinoplastia promoveu melhora nos parâmetros hemogasométricos que indicam acidose respiratória secundária à obstrução das vias aéreas, mostrando-se eficaz no tratamento da síndrome respiratória dos cães braquicefálicos e evidenciando que a hemogasometria arterial é um exame útil no diagnóstico de quadros de acidose respiratória secundária à síndrome dos cães braquicefálicos, sendo indicada no acompanhamento da resposta do paciente ao tratamento.

\section{REFERÊNCIAS}

DiBARTOLA, D.V.M. Introduction to acid-base disorders. In: Fluid, electrolyte and acidbase disorders in small animal practice. St Louis: Saunders Elsevier, 2006. p.229-251.

DUPRÉ, G.; HEIDENREICH, D. Brachycephalic syndrome. Vet. Clin. Small Anim., v.46, p.691-707, 2016.

FOSSUM, T.W.; DUPREY, L.P. Cirurgia do sistema respiratório superior: cirurgia de pequenos animais. 2.ed. São Paulo: Roca, 2005. p.716-758.

HOAREAU, G.L. et al. Evaluation of arterial blood gases and arterial blood pressures in brachycephalic dogs. J. Vet. Intern. Med.,v.26, p.897-904, 2012.

$\mathrm{KOCH}$, D.A. et al. Brachycephalic syndrome in dogs. Compend. Cont. Educ. Pract. Vet., v.25, p.48-55, 2003.

MEOLA, S.D. Brachycephalic airway syndrome. Top. Companion Anim. Med., v.28, p.91-96, 2013.

PACKER, R.M.A.; TIVERS, M.S. Strategies for the management and prevention of conformation-related respiratory disorders in brachycephalic dogs. Vet. Med. Res. Rep., p.219$232,2015$. 
ROELS, E. et al. Comparison of four point-ofcare blood gas analyzers for arterial blood gas analysis in dogs breathing room air. J. Vet. Emerg. Crit. Care, v.3, p.352-359, 2015.

SLAWUTA, P.; GLISKA-SUCHOCKA, K.; CEKIERA, A. The use of elements of the Stewart model (Strong Ion Approach) for the diagnostics of respiratory acidosis on the basis of the calculation of a value of a modified anion gap in brachycephalic dogs. Pol. J. Vet. Sci., v.18, p.217-222, 2015.
SLAWUTA, P.; NICPO`N, J.; DOMANSKA, S. Influence of the wing-of-thenostrils correction procedure on the change of the acid-base balance parameters and oxygen concentration in the arterial blood in French bulldogs. Pol. J. Vet. Sci., v.14, p.77-80, 2011.

TRAPPLER, M.; MOORE, K. Canine brachycephalic airway syndrome: surgical management. Vet. Learn, v.33, p.E1-E8, 2011.

YAMINI, S.H. et al. Estimation of blood gas parameters in Brachycephalic breeds. Int. J. Adv. Mult Res., v.2, p.73-75, 2015. 